\title{
Roof Snow Removal Device Based on Visual Sensing Technology
}

\author{
Zhiyang Zhang ${ }^{1, \text { a }}$ \\ ${ }^{1}$ Department of Mechanical Engineering and Automation, Wuhan University of Technology, Wuhan, Hubei, 430070, China
}

\begin{abstract}
Based on the analysis of the current situation of roof snow removal technology at home and abroad, this paper proposes a fully automatic roof snow removal device based on visual sensing technology. This product is composed of five functional modules: cutting snow removal module, frozen snow assisting removal module, pulley block anti-drop module, worm gear transmission module, and crawler movement module. Through the cooperation of various mechanisms, the efficient removal of snow on the roof is realized. Automatic removal can effectively reduce the adverse impact of snow on the roof on residents' lives and economic development.
\end{abstract}

\section{Research background}

Snow on the roof has always been one of the problems that plagued the residents of our country, especially the residents in the north. Today, most of the housing constructions are made of reinforced concrete truss structures. After a long and intense snowfall, if the roof is covered with snow which is not cleaned up in time, after day and night, it will increase the force on the roof until it reaches the fatigue strength of the steel bars, which will cause immeasurable harm. We can take the northern part of our country as an example. The long winter is always accompanied by heavy snowfall. In some areas, snow disasters may even occur. If the snow is too thick and not cleaned up in time, houses may collapse and the lives of residents may be seriously threatened. At the same time, it is accompanied by heavy property losses, so how to solve the roof snow problem in a timely and efficient manner is extremely urgent.

Traditional snow removal methods rely on manual removal, which is currently the most common household snow removal method in my country. However, manual snow removal is inefficient, labor-intensive, and working environment is limited, so it is no longer suitable for towns with many houses today. Mechanical method is to directly remove the hazard of snow accumulation through mechanical action. At present, the snow removal devices in the world mainly include plow type, high-speed air blowing type, hot melt type, etc. The developed countries have a relatively high degree of mechanization of snow removal and relatively mature technology, but at the same time there are also a series of problems, such as the large size of the machinery, high energy consumption during operation, and the need for professional manipulation.

Our country's research and development of snow removal machinery are relatively late and still in its infancy. In addition, most of the existing professional snow removal devices in our country are aimed at urban roads. The traditional method of removing snow on the roof is still used, that is, a combination of manual and small machinery, but the efficiency is generally low, the labor cost is high, and the use of the effect is not ideal. Therefore, it is necessary to reform and innovate the snow blower, get rid of the traditional large snow blower model, and design a simple, lightweight, highly-automated, and efficient roof snow removal device to solve this problem better.

\section{Overview of the device}

\subsection{Design ideas}

In view of the above research background and market status, we put forward an overall structural design scheme of a fully automatic roof snow removal device based on visual sensing technology based on related literature and actual research. The device is composed of five functional modules, including a cutting snow removal module, an auxiliary snow removal module, a pulley block anti-drop module, a worm gear transmission module, and a crawler motion module. It can realize the functions of removing snow and frozen snow on the roof, and has the characteristics of automation, intelligence, safety and efficiency.

The roof snow removal device is a mechatronic device that integrates mechanical structure and circuit control technology. It first uses mechanical principles, mechanical manufacturing and other disciplines to design the structure, and uses SolidWorks, ANSYS and ADAMS to model and optimize the structure. Afterwards, check and calculate the structure of each part to ensure the rationality and feasibility of the device, and analyze the processing technology to ensure the processing accuracy and

\footnotetext{
a Corresponding author:2978863853@qq.com
} 
assembly requirements. In addition, through the design of the control system, electromechanical integration is realized and the automation level of the device is improved. Finally, the entity is made and debugged. The specific design ideas are as follows:

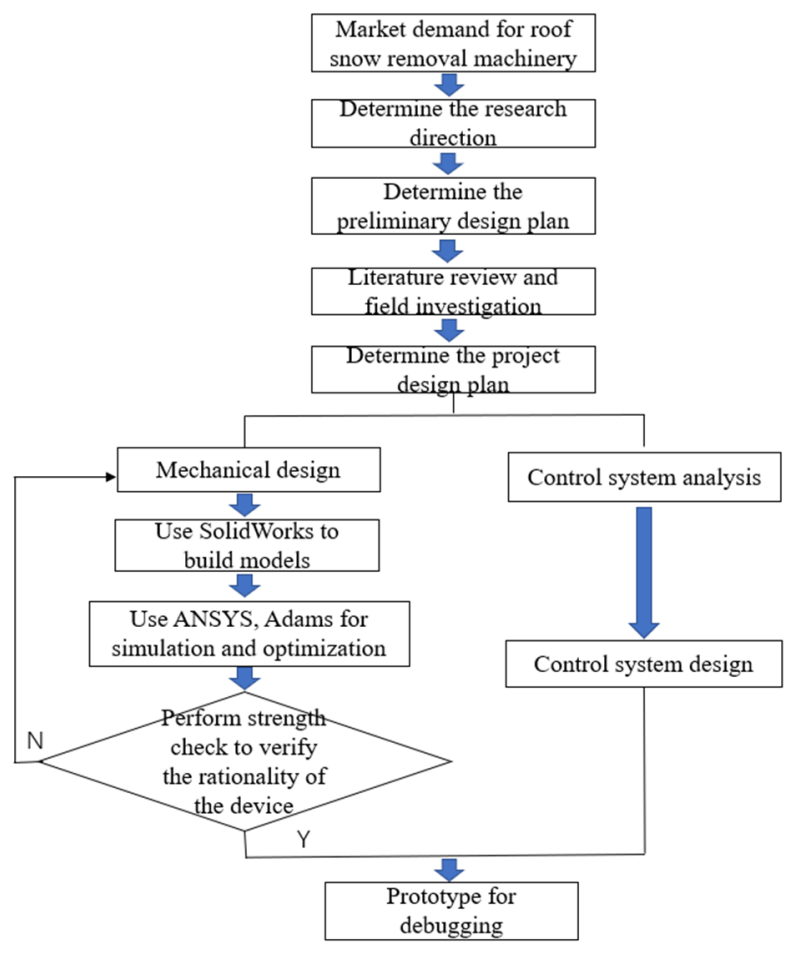

Figure 1 Design idea diagram

\subsection{Overall design}

The whole system is composed of mechanical structure and control system. The mechanical structure mainly includes the auxiliary snow removal device at the rear, the sliding block anti-drop device installed on the upper part, the snow removal device at the lower part of the frame, the worm gear drive and the crawler motion device. The trolley is constructed as the basic frame of the system. The snow removal device uses a single-line path to cut the entire snow layer into small snow blocks to facilitate snow sliding. At the same time, a mechanical structure is used to remove the roof edge and other corners to improve the cleaning effect. Make sure to remove all snow from the roof. Worm gear drives use electric motors to drive worms to rotate. The worm is located above the snow removal device. The rotation of the worm can drive the chip snow removal device to move horizontally and longitudinally, so when the trolley is in a blind area (such as the end of the roof), the device can drive the chi to clear snow. When the trolley encounters high hardness of frozen snow and cannot perform the cutting task, the auxiliary snow removal module starts to work, the motor drives the cutter to rotate to break the icy snow, and then the cutting snow removal device continues to complete the subsequent cutting work and realize the installation cooperation, and achieve a good snow removal effect. The working principle of the sliding block anti-drop module is that the guide rail fixed on the roof is connected with the pulley fixed on the trolley through a rope. The device can prevent the trolley from falling from the roof, ensure the safety of the snow removal trolley, and avoid accidental injury to pedestrians on the ground. The trolley can provide a certain amount of traction to reduce the difficulty when the trolley encounters greater resistance and is difficult to move. The product relies on mechanical structure and circuit control technology to effectively remove snow on the roof and ensure efficient and safe work. This is a mechatronics device that integrates multiple functions.

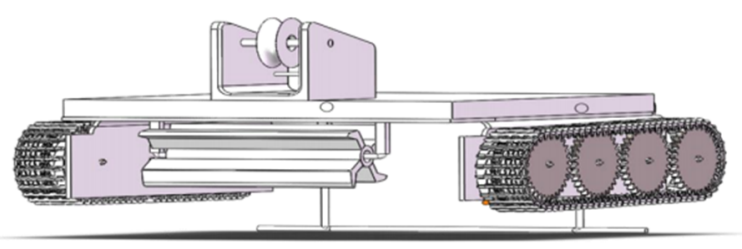

Figure 2 The overall structure of the roof snow blower

\subsection{Device design plan for each module}

\subsubsection{Cutting snow removal module}

The snow removal module includes a bottom plate, telescopic rod, plastic film, motor, worm, etc. Fig. 3 is a three-dimensional schematic diagram of the cutting snow removal module. The worm rotates under the drive of the motor, and is driven by the worm gear, and the screw rotates. The vertical telescopic rod can adapt the trolley to snow work of different thickness, and the telescopic rods at both ends can adjust the cutting area to improve the snow removal efficiency. The roof snow removal trolley moves, the telescopic rod cuts the snow from both sides, and the cut snow moves onto the plastic film. As the plastic film reduces friction, the snow slides off the roof under the action of gravity. When moving to the top of the roof, the trolley stops, and the snow-cutting device moves relatively to the trolley to cut the snow and remove the snow at the blind spots. The simulation diagram of the working process of the cutting snow removal module is shown in Figure 4.

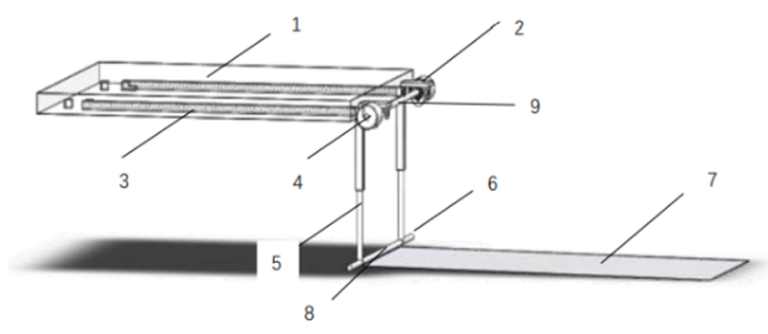

Figure 3 Cutting snow removal module 


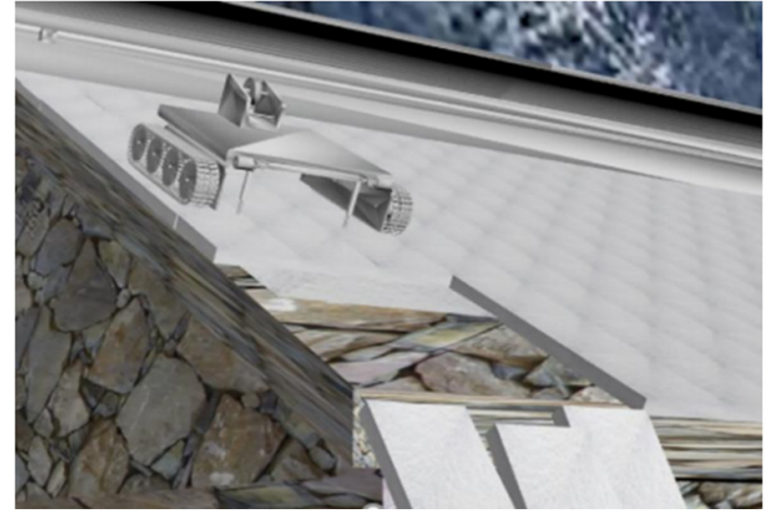

Figure 4 Schematic diagram of cutting snow removal device

After the snow is cut, it will slide down the roof under its own gravity, which can reduce the energy consumption of the machine. The feasibility of this method is verified by calculation. Figure 5 is a schematic diagram of the force on the snow block. $G$ is the gravity of the snow block, $F$ represents the component force of gravity along the slope of the roof, $F_{N}$ is the supporting force of the roof, $F_{f}$ is the friction force of the plastic film, and $\alpha$ is the inclination angle of the roof.

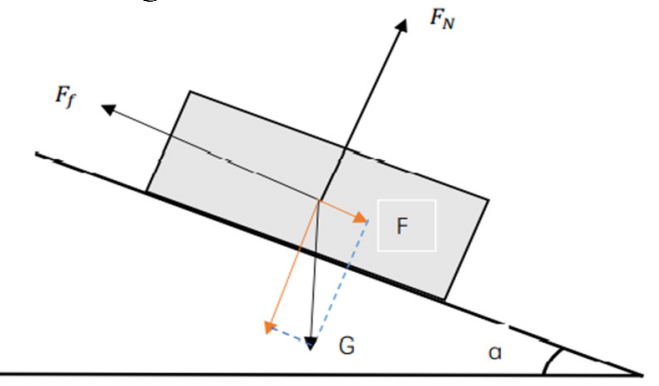

Figure 5 Schematic diagram of the force on the snow block

$$
\begin{gathered}
F_{f}=\mu F_{N}=\mu m g \cos \alpha \\
F=m g \sin \alpha
\end{gathered}
$$

By consulting the data, we can get: the friction coefficient of PTFE plastic film $\mu=0.04$. In order to make the snow block fall smoothly, it needs to meet:

$$
F>F_{f}
$$

Solving simultaneous equations, we can get:

$$
\alpha>2.29^{\circ} \text {. }
$$

From the above force analysis, it can be seen that when the roof inclination angle is greater than $2.29^{\circ}$, the snow block can slide down smoothly. Generally, the roof inclination angle of houses in China is $30^{\circ}$, so this device is feasible and can be widely used in different situations.

\subsubsection{Frozen snow auxiliary removal module}

The auxiliary frozen snow removal module is mainly composed of cutters, telescopic rods and motors. The cutter is installed at the lower part of the telescopic rod and rotated by the motor. When the trolley encounters frozen snow and its movement is blocked, it will stop moving forward under the control of the control system, and then control the telescopic rod to extend. When the cutter just touches the frozen snow, the motor drives the motor to rotate quickly to break the frozen snow. At the same time, the telescopic rods slowly extend and coordinate with each other to completely break the frozen snow. When the removal is completed, the motor stops rotating, the cutter stops rotating, and the telescopic rod retracts to the original height, and the cutting snow removal device is started again to remove.

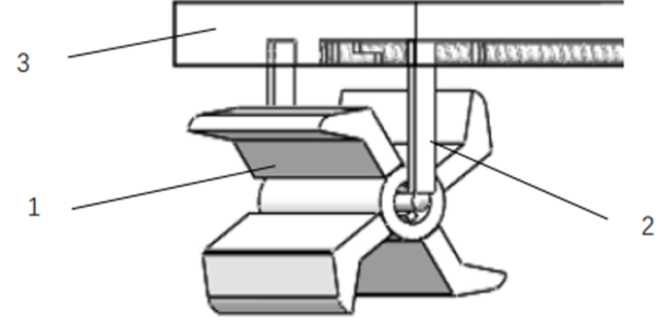

Figure 6 Frozen snow auxiliary removal module

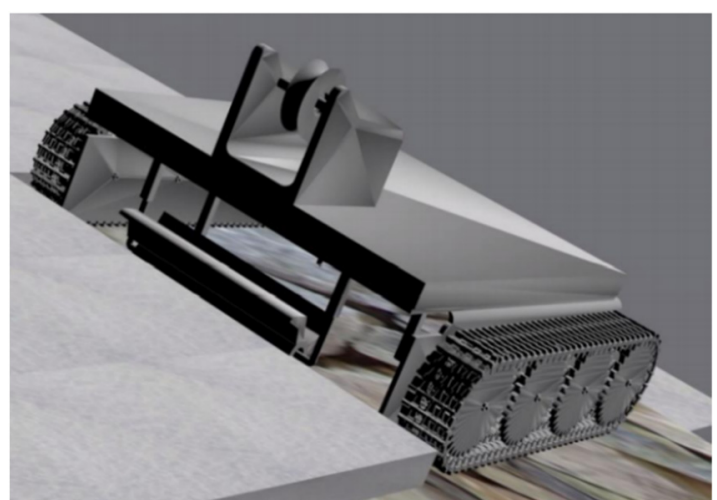

Figure 7 Schematic diagram of the frozen snow auxiliary removal device

\subsubsection{Worm gear drive module}

Worm gears include motor, worm, screw and other components. The worm is fixed to ensure the stability of the work. The left and right motors work at the same time to drive the worm to rotate, and then the meshing drive drives the screw to rotate. Since the left and right screw are driven by the same worm, they rotate synchronously, making the installation the telescopic rod on the screw rod moves synchronously. When the trolley moves to the top of the roof, that is, when the blind spot is cleared, the worm drives the snow removal device installed on the screw to move relatively to the trolley to achieve the purpose of clearing the snow at the end. Figure 8 is a threedimensional model diagram of a worm gear transmission.

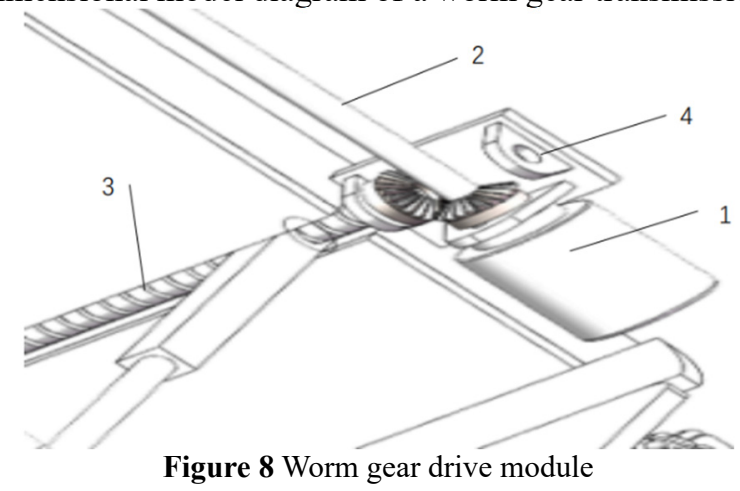

Figure 8 Worm gear drive module 


\subsubsection{Pulley slider anti-drop module}

The sliding block anti-drop device is composed of a guide rail fixed on the roof, a pulley fixed on the trolley, and a rope. The guide rail is connected with the pulley through a rope to provide pulling force to the trolley to ensure that the trolley will not fall from the roof during snow removal, thereby ensuring the normal operation of the device and the safety of pedestrians under the roof. At the same time, when the trolley is cleaning the thick snow layer, the movement resistance is relatively large, and the power provided by the crawler is insufficient. At this time, the motor on the guide rail can shrink the rope by rotating, thereby providing a certain traction to pull the trolley to move. Figure 9 is a diagram of the pulley slider anti-drop module.

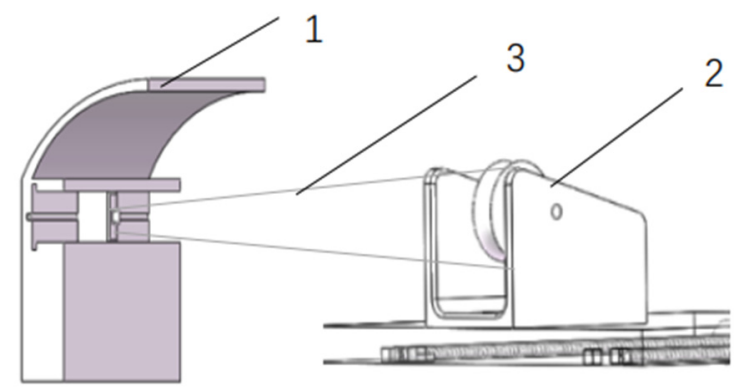

Figure 9 Pulley slider anti-drop module

\subsubsection{Crawler motion module}

The crawler motion module is composed of gears, tracks and motors, which ensures the most basic plane movement of the roof snow removal trolley. The use of crawlers allows the trolley to adapt to the unevenness of the roof, and at the same time increases the contact area between the trolley and the snow, thereby reducing the pressure and ensuring that the device will not be trapped in the snow and hinder normal operation. Figure 10 is a threedimensional schematic diagram of a crawler motion module.

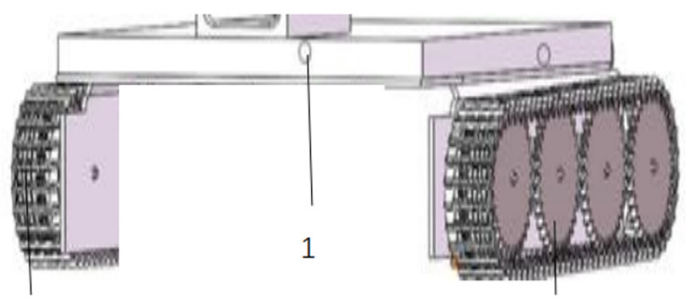

Figure 10 Crawler motion module

\subsubsection{Control system design}

The control system is mainly embodied in the crawler motion control module, frozen snow assisted removal control module and the worm gears drive control module, and is completed with the help of visual sensors and pressure sensors. The visual sensor is used to judge the distance between the trolley and the top of the roof. When it moves to the position closest to the top, the information is transmitted to the control system in time, so that the trolley stops moving accurately. Then the worm gear control system starts to work and controls the rotation of the motor. When the specified number of turns is rotated (that is, the cutting snow removal device has completed the removal of snow on the top of the roof), the motor starts to rotate the same number of turns in reverse, the purpose is to drive the cutting snow removal device back to its original position. The pressure sensor can monitor the changes in the resistance of the trolley movement. When the resistance suddenly increases, such as frozen snow, the mechanical sensor transmits the information to the control system to control it to stop moving and control the operation of the frozen snow auxiliary removal device. After the frozen snow is cleared, the pressure sensor feels the resistance reduction, and transmits this information to the control system to control the shortening of the telescopic rod, then the motor stops rotating, and the entire frozen snow removal process is completed. The relationship of the control system is shown in Figure 11.

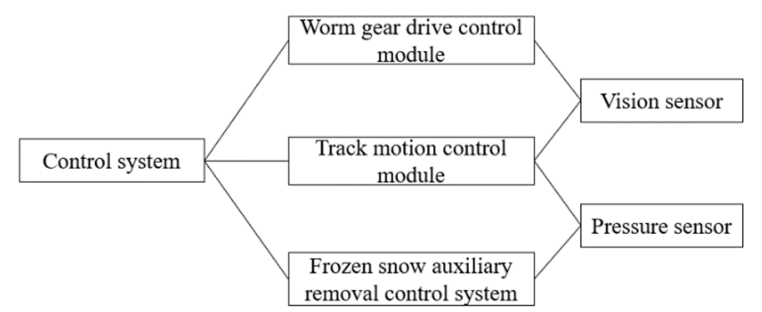

Figure 11 Schematic diagram of control system relationship

\section{Conclusion}

With the development of science and technology, the future roof snow removal equipment will gradually develop toward automation and intelligence to meet people's needs better. This product, the roof snow removal device based on visual sensing technology, is a mechatronic device integrating mechanical structure and circuit control technology. It has the advantages of novel structure, strong practicability, and high level of automation. It is believed that with the progress of society and the increase in market demand, there will be a broader space for this device to develop in the future.

\section{References}

1. Zhen Wang. (2003) Discussion on the status quo of domestic snow and ice removal machinery. Construction machinery, 34(4):55-57.

2. Xianjiang Cui. (1988) Snow Removal Machinery. China Communications Press, Beijing.

3. Heng Sun, Zuomo Chen, Wenjie Ge. (2006) Principles of Machinery (Seventh Edition). Higher Education Press, Beijing.

4. Jingmin Sun, Yingchun Liang. (2009) Mechanical Optimization Design (fourth edition)Machinery Industry Press, Beijing.

5. Nianxin Xiao, Changrong Liu. (2006) Theoretical mechanics. China Agricultural Science and Technology Press. Beijing.

6. Changrong Liu, Yucai Zheng. (2002) Fundamentals of Mechanical Design. China Agricultural Science and Technology Press. Beijing. 